\title{
Design of Second-Order Sliding Mode Guidance Law Based on the Nonhomogeneous Disturbance Observer
}

\author{
Huibo Zhou, ${ }^{1,2}$ Shenmin Song, ${ }^{1}$ Junhong Song, ${ }^{1}$ and Jing Niu ${ }^{2}$ \\ ${ }^{1}$ Center for Control Theory and Guidance Technology, Harbin Institute of Technology, Harbin 150001, China \\ ${ }^{2}$ School of Mathematical Sciences, Harbin Normal University, Harbin 150009, China \\ Correspondence should be addressed to Huibo Zhou; zhouhb0306@sina.com
}

Received 17 March 2014; Accepted 26 August 2014; Published 22 September 2014

Academic Editor: Zoltan Szabo

Copyright (c) 2014 Huibo Zhou et al. This is an open access article distributed under the Creative Commons Attribution License, which permits unrestricted use, distribution, and reproduction in any medium, provided the original work is properly cited.

\begin{abstract}
Considering the guidance problem of relative motion of missile target without the dynamic characteristics of missile autopilot in the interception planar, non-homogeneous disturbance observer is applied for finite-time estimation with respect to the target maneuvering affecting the guidance performance. Two guidance laws with finite-time convergence are designed by using a fast power rate reaching law and the prescribed sliding variable dynamics. The nonsingular terminal sliding mode surface is selected to improve dynamic characteristics of missile autopilot. Furthermore, the finite-time guidance law with dynamic delay characteristics is designed for the target maneuvering through adopting variable structure dynamic compensation. The simulation results demonstrate that, for different target maneuvering, the proposed guidance laws can restrain the sliding mode chattering problem effectively and make the missile hit the maneuvering target quickly and accurately with condition of corresponding assumptions.
\end{abstract}

\section{Introduction}

With the development of flight control technology, the maneuverability of targets is getting much stronger, and how to inhibit the influence of the target's maneuvering on the missile's guidance performance and improve the robustness of guidance law is always the research hotspot in the field of missile interception. At present, there are several robust guidance laws, including $H_{\infty}$ guidance law [13], $L_{2}$ gain guidance law [4], neural network guidance law [5], adaptive guidance law [6], and sliding mode variable structure guidance law. The sliding mode guidance law which is robust with respect to uncertainties and disturbances has aroused a wide research interest in recent years [7-11].

However, there is a disadvantage for the sliding mode variable structure control, which is the chattering after the system reaches the sliding mode manifold. Currently, the main solutions to this disadvantage are high-order sliding mode control [10-12] and nonsingular terminal sliding mode control [13]. The higher order sliding mode is an extension of the conventional sliding mode and it cannot only eliminate the defect of the conventional sliding mode method but also maintain its advantages. Because the second-order sliding mode controller has simple structure and needs less information, it is the most widely used in the higher order sliding mode.

Nonsingular terminal sliding mode can remove the chattering and allow the system state to converge to the equilibrium within finite time. However, considering the capability to reach the sliding mode, this speed is too slow compared to exponential reaching law, even at the space adjacent to the sliding mode with fast exponential terminal sliding mode control; therefore, nonsingular terminal sliding mode with the advantages of no chattering has lower comprehensive control performance. Yu et al. combined the traditional power reaching law and exponential reaching law to get a fast power reaching law, which solves the problems of the traditional power reaching law [14]. Considering this advantage, this paper combines the nonsingular terminal sliding mode and fast power reaching law, which both remove the chattering problem of sliding mode and improve the speed to reach the sliding mode.

During the missile's terminal guidance, a main factor affecting its precision is the target's escape maneuvering. For 
this problem, the target's maneuvering with an extended state observer independent of system model was proposed in [15]; despite of its high accuracy, this simulation has no stable theoretical proofs. An observer that can estimate unknown disturbance precisely was researched by [16-18]; however, the result is not finite-time convergent. A homogeneous disturbance observer was studied in [11], which is able to perform transient estimation with high precision of the target's maneuvering in the system. However, this method converges quite slowly when there is great initial error, which is determined by the nature of homogeneous system. A nonhomogeneous and finite-time convergent robust differentiator was proposed by [19], based on which, a nonhomogeneous disturbance observer to speed up the transient process in finite time was researched by [20].

The dynamic delay characteristic of missile autopilot is another major factor influencing the guidance precision in the process of interception guidance within the atmosphere. In order to deal with this problem, the guidance loop and control loop are studied as a whole. The method which can work in the best of maneuvering abilities and strike the target with high precision has become an inevitable trend of missile guidance and control system design [2125]. The guidance laws with the dynamic delay characteristic are considered by using the differential game and optimal control theory in $[21,22]$; however, the remaining time for the guidance needs to be estimated. The guidance law with attack angle constraints and dynamic delay is researched by using observer in $[23,24]$, but the selected sliding mode surface is too complex. Using back-stepping method, a guidance law considering the missile's dynamic characteristics was studied in [25], but this control method need a great deal of computation.

Inspired by the literatures above, this paper develops three finite-time guidance laws. Not considering the dynamic delay of missile autopilot, two guidance laws with finitetime convergence are designed by using a fast power rate reaching law and the prescribed sliding variable dynamics. A nonhomogeneous disturbance observer is applied to perform finite-time observation to the target's disturbance in missile guidance system. Considering the missile's dynamic characteristics, this paper designs a guidance law combining nonsingular terminal sliding and fast power rate reaching law. It is able to compensate the missile's dynamic delay and the target's maneuvering, mitigate the chattering, shorten the time before reaching the sliding surface, and improve the system's disturbance resistance and the precision of guidance.

\section{General Description}

Considering the relative motion geometry of missile and target in the intercepting plane, both of which are regarded as point masses, their connecting line is the line-of-sight (LOS), as shown in Figure 1. $M$ and $T$ represent the missile and the target, respectively.

During the attack, the impact angle is quite small, and differential equations can be derived from Figure 1 as follows:

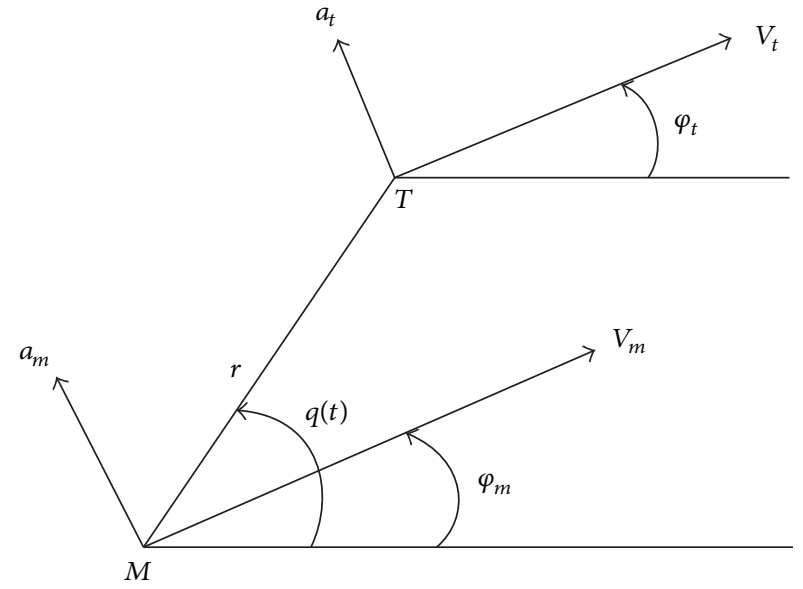

FIGURE 1: Relative motion geometry of missile and target.

$$
\begin{aligned}
\dot{r} & =V_{t} \cos \left(q-\varphi_{t}\right)-V_{m} \cos \left(q-\varphi_{m}\right), \\
r \dot{q} & =-V_{t} \sin \left(q-\varphi_{t}\right)+V_{m} \sin \left(q-\varphi_{m}\right),
\end{aligned}
$$

where $V_{m}$ and $V_{t}$ are the velocities of the target and missile, respectively; $r$ and $\dot{r}$ are the relative distance between the missile and the target and the relative speed, in which $r$ and $\dot{r}$ are regarded as known time-varying parameters; $q, \dot{q}$ are the LOS angle and the LOS angular rate, respectively. $\varphi_{m}, \varphi_{t}$ are the missile's and the target's flight path angles, respectively.

Taking the first-order derivatives of both sides of (2) with respect to time yields

$$
\ddot{q}=-\frac{2 \dot{r}}{r} \dot{q}-\frac{\cos \theta_{m}}{r} a_{m}+\frac{\cos \theta_{t}}{r} a_{t},
$$

where $\theta_{m}=q-\varphi_{m}, \theta_{t}=q-\varphi_{t}$ are advance angles of missile and target, respectively; $a_{m}$ and $a_{t}$ the missile's and the target's normal accelerations, respectively. During designing the guidance law, the latter is regarded as the system's control input and unknown external disturbance input, respectively.

For convenience of designing the guidance law, the following assumptions are given.

Assumption 1. As is restrained by acceleration capability, the maximum lateral acceleration that can be actually provided by the missile and the target is limited; therefore, there exist constants $A_{m}>0, A_{1}>0$, and $A_{2}>0$ which allow

$$
\left|a_{m}\right| \leq A_{m}, \quad\left|a_{t}\right| \leq A_{1}, \quad\left|\dot{a}_{t}\right| \leq A_{2} .
$$

During terminal guidance, as is restrained by the power of its angle tracing system, receiver acceleration, and other factors, the seeker has a minimum operating range $r_{0}$. When the relative distance between the missile and the target is no more than $r_{0}$, the guidance circuit is broken; therefore, a guidance process satisfying the hypothesis below is required.

Assumption 2 (see [18]). The time-varying parameter $r(t)$ in the system of (2) satisfies

$$
r(t) \geq r_{0}
$$




\section{Prerequisite Knowledge}

3.1. Nonhomogeneous Disturbance Observer. Consider firstorder SISO nonlinear system:

$$
\dot{s}=g(t)+u \quad s \in R .
$$

Equation (6) describes the dynamic characteristics of the sliding mode along the trajectory of the system. $s=0$ defines the system's motion on the sliding mode manifold, $u \in R$ is the continuous control input, and $g(t)$ is a sufficiently smooth indeterminate function.

If the sliding mode variable $s$ and the control input $u$ can be obtained in real time, $g(t)$ is $m-1$ order differentiable, and $g^{m-1}(t)$ has a known Lipschitz constant $L$. A nonhomogeneous disturbance observer is designed to speed up the transient process in [20], which is defined as

$$
\begin{aligned}
& \dot{z}_{0}=v_{0}+u, \quad v_{0}=h_{0}\left(z_{0}-s\right)+z_{1}, \\
& \dot{z}_{1}=v_{1}, \quad v_{1}=h_{1}\left(z_{1}-v_{0}\right)+z_{2}, \\
& \quad \vdots \\
& \dot{z}_{m-1}=v_{m-1}, \quad v_{m-1}=h_{m-1}\left(z_{m-1}-v_{m-2}\right)+z_{m}, \\
& \dot{z}_{m}=h_{m}\left(z_{m}-v_{m-1}\right),
\end{aligned}
$$

where $h_{i}$ is a function with a form as follows:

$$
h_{i}(\sigma)=-\lambda_{m-i} L^{1 /(m-i+1)}|\sigma|^{(m-i) /(m-i+1)} \operatorname{sgn}(\sigma)-\mu_{m-i} \sigma,
$$

where $\lambda_{i}, \mu_{i}>0, i=0,1, \ldots, m$.

Compared with the observer in Shtessel et al. [11], $h_{i}(\sigma)$ which is defined as (8) has an additional linear condition $\mu_{m-i} \sigma$ to speed up the transient process.

Lemma 3 (see [20]). Suppose that $s(t)$ and $u(t)$ in the system of (6) are measured; the parameters $\lambda_{i}$ and $\mu_{i}$ are chosen sufficiently large in the reverse order. The following equalities are true after a finite time of the transient process:

$$
\begin{gathered}
z_{0}=s(t), \quad z_{1}=g(t), \ldots, \\
z_{i}=v_{i-1}=g^{(i-1)}, \quad i=1, \ldots, m .
\end{gathered}
$$

3.2. Fast Power Reaching Law. The fast power reaching law is described by a first-order nonlinear equation:

$$
\dot{s}=-k_{1}|s|^{\alpha} \operatorname{sgn}(s)-k_{2} s,
$$

where $s \in R$ is the sliding mode variable, $k_{1}, k_{2}>0, \alpha \in(0,1)$, and $\operatorname{sgn}(\cdot)$ is the sign functions.

For convenience, we denote $\operatorname{sig}(s)^{\alpha}=|s|^{\alpha} \operatorname{sgn}(s)$. It can be verified that

$$
\begin{aligned}
& \frac{d}{d t}|s|^{\alpha+1}=(\alpha+1) \operatorname{sig}(s)^{\alpha}, \\
& \frac{d}{d s} \operatorname{sig}(s)^{\alpha+1}=(\alpha+1)|s|^{\alpha} .
\end{aligned}
$$

Lemma 4 (see [14]). For the system of (10), if $k_{1}, k_{2}>0$ and $\alpha \in(0,1)$, the system state $s$ and its first-order derivative $\dot{s}$ will tend to 0 within finite time $T$, and the time of adjustment $T$ will be a continuous function at the initial condition:

$$
T(s(0))=\frac{\ln \left(1+\left(k_{2} / k_{1}\right)|s(0)|^{1-\alpha}\right)}{k_{2}(1-\alpha)} .
$$

\subsection{Finite-Time Convergence}

Definition 5 (see [26]). The equilibrium $x=0$ of the system is finite-time stable if it is asymptotically stable with a finite settling time for any solution and initial conditions.

Definition 6 (see [27]). Consider a smooth dynamic system $\dot{x}=v(x)$ with a smooth output function $s(t, x)=0$. Then provided that successive total time derivatives $s, \dot{s}, \ddot{s}, \ldots, s^{(r-1)}$ are continuous functions of the closed system state variables, and the $r$-sliding point set (13) is nonempty and consists locally of Filippov trajectories, the motion on set (13) is called $r$-sliding mode.

Lemma 7 (see [14]). Considering a nonlinear system of $\dot{x}=$ $f(x, t), f(0, t)=0, x \in R^{n}$, if there is a continuous and positive definite function $V(t)$ satisfying the differential inequality as follows:

$$
\dot{V}(x)+k_{2} V(x)+k_{1} V^{\alpha}(x) \leq 0,
$$

where $k_{1}, k_{2}>0,0<\alpha<1$ are all constants, the time $T$ for the system state to reach the stable point satisfies the inequality below:

$$
T \leq \frac{1}{k_{2}(1-\alpha)} \ln \frac{k_{2} V^{1-\alpha}\left(x_{0}\right)+k_{1}}{k_{1}} .
$$

\section{Guidance Law Design}

Define $x=\dot{q}$, and system equation (3) can be written as

$$
\dot{x}=-\frac{2 \dot{r}}{r} x-\frac{a_{m} \cos \theta_{m}}{r}+\frac{a_{t} \cos \theta_{t}}{r} .
$$

Let

$$
a_{m}=\frac{-2 \dot{r} x-r u}{\cos \theta_{m}}, \quad g(t)=\frac{a_{t} \cos \theta_{t}}{r} .
$$

When substituting (16) into (15), we can get

$$
\dot{x}=u+g(t) .
$$

Select the sliding mode manifold:

$$
s=x, \quad \dot{s}=g(t)+u .
$$

Selecting (10) of fast power reaching law and combining (17) and (18), we can get

$$
u=-k_{1} \operatorname{sig}(s)^{\alpha}-k_{2} s-g(t),
$$


where $g(t)=a_{t} \cos \theta_{t} / r$ is the unknown total disturbance of the target, and

$$
\begin{aligned}
|\dot{g}(t)|=\mid- & \frac{a_{t} \sin \theta_{t}\left(V_{m} \sin \theta_{m}-V_{t} \sin \theta_{t}\right)}{r^{2}} \\
& -\frac{a_{t} \cos \theta_{t}\left(V_{t} \cos \theta_{t}-V_{m} \cos \theta_{m}\right)}{r^{2}} \\
& +\frac{\dot{a}_{t} \cos \theta_{t}}{r}+\frac{a_{t}^{2} \sin \theta_{t}}{r V_{t}} \mid \\
\leq & \frac{A_{2}}{r_{0}}+\frac{2 A_{1}\left(V_{m}+V_{t}\right)}{r_{0}^{2}}+\frac{A_{1}^{2}}{r_{0} V_{t}} \\
= & L .
\end{aligned}
$$

Case 1. Suppose that the interceptor missile autopilot has ideal dynamics.

For the system of (18), the nonhomogeneous disturbance observer introduced by (7) and (8) is adopted to estimate $g(t)$. Let $m=2$ :

$$
\begin{aligned}
& \dot{z}_{0}=v_{0}-\frac{2 \dot{r}}{r} \dot{q}-\frac{a_{m} \cos \theta_{m}}{r}, \\
& v_{0}=-\lambda_{2} L^{1 / 3}\left|z_{0}-s\right|^{2 / 3} \operatorname{sgn}\left(z_{0}-s\right)-\mu_{2}\left(z_{0}-s\right)+z_{1}, \\
& \dot{z}_{1}=v_{1}, \\
& v_{1}=-\lambda_{1} L^{1 / 2}\left|z_{1}-v_{0}\right|^{1 / 2} \operatorname{sgn}\left(z_{1}-v_{0}\right)-\mu_{1}\left(z_{1}-v_{0}\right)+z_{2}, \\
& \dot{z}_{2}=-\lambda_{0} L \operatorname{sgn}\left(z_{2}-v_{1}\right)-\mu_{0}\left(z_{2}-v_{1}\right) .
\end{aligned}
$$

According to Lemma 3, after the system's transient process in finite time, $g(t)=z_{1}$, we can design the guidance law as

$$
a_{m}=\frac{-2 \dot{r} x-r\left(-k_{1} \operatorname{sig}(s)^{\alpha}-k_{2} s-z_{1}\right)}{\cos \theta_{m}} .
$$

Theorem 8. For the state equation of system (15) under the influence of the designed nonhomogeneous disturbance observer of (21), and after the transient process in finite time for the disturbance of the target in the system, $g(t)=z_{1}$, the $L O S$ angular rate and the LOS angular acceleration in (15) can converge to zero within finite time by designing the guidance law of (22) and dynamic compensation.

Proof. Substituting the guidance law of (22) into the system of (15), we can get

$$
\begin{aligned}
\dot{x}= & -\frac{\cos \theta_{m}}{r}\left[\frac{-2 \dot{r} x-r\left(-k_{1} \operatorname{sig}(s)^{\alpha}-k_{2} s-z_{1}\right)}{\cos \theta_{m}}\right] \\
& -\frac{2 \dot{r}}{r} x+\frac{a_{t} \cos \theta_{t}}{r} \\
= & -k_{2} s-k_{1} \operatorname{sig}(s)^{\alpha}-z_{1}+\frac{a_{t} \cos \theta_{t}}{r},
\end{aligned}
$$

where $s=x, a_{t} \cos \theta_{t} / r=g(t)$, and $g(t)=z_{1}$ after the transient process in finite time according to the observer of (21); thus,

$$
\dot{s}=-k_{1}|s|^{\alpha} \operatorname{sgn}(s)-k_{2} s .
$$

According to Lemma 4, the system state $s$ and its firstorder derivative $\dot{s}$ converge to zero within finite time $T$, which means that the LOS angular rate and the LOS angular acceleration in the system of (15) converge to zero within finite time.

For convenience, the guidance law designed with (22) is called the fast sliding mode guidance law, or FSMG for short.

For the system of $\dot{s}=g(t)+u$, Shtesel et al. proposes a sliding mode control method with compensated $s$-dynamics [28]. Inspired by this method, we use the observer designed with (21) to estimate unknown disturbance $g(t)$ and to design the guidance law $\widetilde{a}_{m}$ :

$$
\begin{aligned}
\widetilde{a}_{m} & =\frac{-2 \dot{r} \dot{q}-r u}{\cos \theta_{m}}, \\
v & =-a_{1}|s|^{2 / 3} \operatorname{sgn}(s)+w-z_{1}, \\
\dot{w} & =-a_{2}|s|^{1 / 3} \operatorname{sgn}(s),
\end{aligned}
$$

where $a_{1}>0, a_{2}>0$,

Theorem 9. For the guidance law of (25), $z_{1}$ is the target's disturbance estimated with nonhomogeneous disturbance observer of (21) after finite time; then this guidance law allows $s, \dot{s}$ in the state equation of system (15) to converge to zero within finite time, which means that the LOS angular rate and the LOS angular acceleration can converge to zero within finite time.

Proof. Substituting the guidance law of (25) into the system of (18), we can get

$$
\begin{aligned}
\dot{s} & =g(t)+u \\
& =g(t)-a_{1}|s|^{2 / 3} \operatorname{sgn}(s)+w-z_{1}, \\
\dot{w}= & -a_{2}|s|^{1 / 3} \operatorname{sgn}(s) .
\end{aligned}
$$

Under the action of nonhomogeneous disturbance observer of (21), the form we get is

$$
\begin{aligned}
& \dot{s}=-a_{1}|s|^{2 / 3} \operatorname{sgn}(s)+w, \\
& \dot{w}=-a_{2}|s|^{1 / 3} \operatorname{sgn}(s) .
\end{aligned}
$$

Construct a Lyapunov function:

$$
V_{1}=\frac{3}{4} a_{2}|s|^{4 / 3}+\frac{1}{2} \omega^{2}
$$


It is not hard to verify that $V_{1}(t)$ is positive definite, continuous, and differentiable. Taking the derivative of (28), we can get

$$
\begin{aligned}
\dot{V}_{1}(t)= & a_{2}|s|^{1 / 3} \operatorname{sgn}(s) \dot{s}+w \dot{w} \\
= & a_{2}|s|^{1 / 3} \operatorname{sgn}(s)\left(-a_{1}|s|^{2 / 3} \operatorname{sgn}(s)+w\right) \\
& +w\left(-a_{2}|s|^{1 / 3} \operatorname{sgn}(s)\right) \\
= & -a_{1} a_{2}|s| \\
\leq & 0 .
\end{aligned}
$$

According to LaSalle principle, $\dot{V}_{1}(t)=0$ contains a unique solution $s=x=0$; therefore, the system of (27) converges to zero asymptotically. It is obvious that the system of (27) is a homogeneous system, and the degree of homogeneity is -1 according to the theory of homogeneity [11]; therefore, in the system of (27), s, $\dot{s}$ becomes stable at the point of zero within finite time, which means that the LOS angular rate and the LOS angular acceleration can converge to zero within finite time.

For convenience, the guidance law designed with (25) is called the variable dynamic sliding mode guidance law, or VDSMG for short.

Remark 10. For any system with the form $\dot{s}=g(t)+u$, both FSMG and VDSMG are able to ensure that the system's states $s$ and $\dot{s}$ converge to zero in finite time. FSMG is superior to VDSMG with respect to convergence time; meanwhile, the guidance law VDSMG will oscillate between positive and negative values until the sliding mode manifold and its derivative converge to zero. See Section 5 simulation for the detailed comparison.

Remark 11. Both FSMG and VDSMG are designed while not considering the dynamic characteristics of missile autopilot; thus, the precision of guidance is not necessarily guaranteed when there is dynamic delay of autopilot.

It is necessary to design a guidance law with high precision and fast convergence considering the dynamic characteristics of the missile autopilot.

Case 2. Suppose that the interceptor missile autopilot has first-order dynamic delay.

The dynamics of the missile autopilot is described with the first-order inertia link as

$$
\dot{a}_{m}=-\frac{1}{\tau} a_{m}+\frac{1}{\tau} a_{m c}
$$

where $\tau$ is the time constant of missile autopilot, $a_{m c}$ is the guidance command acceleration given to missile autopilot, and $a_{m}$ is the missile's acceleration obtained.

For the system's equations of states (15) and (30), we select the nonsingular terminal sliding mode:

$$
s_{1}=s+\beta \operatorname{sig}(\dot{s})^{\gamma},
$$

where $1<\gamma<2, \beta>0$ are constants.
Select fast power reaching law:

$$
\dot{s}_{1}=-k_{1}\left|s_{1}\right|^{\alpha} \operatorname{sgn}\left(s_{1}\right)-k_{2} s_{1},
$$

where the parameters are the same as those of (10).

Take the derivative of (31):

$$
\begin{aligned}
\dot{s}_{1}=\dot{s}+\beta \gamma|\dot{\boldsymbol{s}}|^{\gamma-1} \ddot{\boldsymbol{s}} & \\
=\dot{s}+\beta \gamma|\dot{s}|^{\gamma-1} \frac{d}{d t} & \left(-\frac{2 \dot{r} x}{r}-\frac{a_{m} \cos \theta_{m}}{r}+g(t)\right) \\
=\dot{s}+\beta \gamma|\dot{s}|^{\gamma-1}( & -\frac{2 \ddot{r} x+2 \dot{r} \dot{x}}{r}+\frac{a_{m} \dot{r} \cos \theta_{m}}{r^{2}} \\
& +\frac{2 \dot{r}^{2} x}{r^{2}}+\frac{a_{m} \dot{q} \sin \theta_{m}}{r}-\frac{a_{m}^{2} \sin \theta_{m}}{r V_{m}} \\
& \left.-\frac{\cos \theta_{m}}{r \tau} a_{m c}+\frac{a_{m} \cos \theta_{m}}{r \tau}+\dot{g}(t)\right) .
\end{aligned}
$$

Combining (32) and (33), we can get

$$
\begin{aligned}
a_{m c}=\frac{r \tau}{\cos \theta_{m}}( & \beta^{-1} \gamma^{-1}|\dot{s}|^{2-\gamma} \operatorname{sgn}(\dot{s})-\frac{2 \ddot{r} s+2 \dot{r} \dot{s}}{r} \\
& +\frac{2 \dot{r}^{2} x}{r^{2}}+\frac{a_{m} \dot{r} \cos \theta_{m}}{r^{2}}+\frac{a_{m} \dot{q} \sin \theta_{m}}{r} \\
& -\frac{a_{m}^{2} \sin \theta_{m}}{r V_{m}}+\frac{a_{m} \cos \theta_{m}}{r \tau} \\
& \left.+\beta^{-1} \gamma^{-1}|\dot{s}|^{1-\gamma}\left(k_{1} \operatorname{sig}\left(s_{1}\right)^{\alpha}+k_{2} s_{1}\right)+\dot{g}(t)\right) .
\end{aligned}
$$

During the terminal guidance, we suppose that $\dot{r}=$ const and $\ddot{r} \approx 0$ basing on the truth that $\dot{r}$ almost remains the same. Meanwhile, as $1-\gamma<0$, the term $\beta^{-1} \gamma^{-1}|\dot{s}|^{1-\gamma}$ becomes singular when $\dot{s} \rightarrow 0$.

Design a guidance law with the dynamic delay of missile autopilot:

$$
\begin{aligned}
\tilde{a}_{m c}= & \frac{r \tau}{\cos \theta_{m}} \\
& \times\left(\beta^{-1} \gamma^{-1} \operatorname{sig}(\dot{s})^{2-\gamma}-\frac{2 \dot{r} \dot{s}}{r}+\frac{2 \dot{r}^{2} x}{r^{2}}\right. \\
& +\frac{a_{m} \dot{r} \cos \theta_{m}}{r^{2}}+\frac{a_{m} \dot{q} \sin \theta_{m}}{r}-\frac{a_{m}^{2} \sin \theta_{m}}{r V_{m}} \\
& \left.+\frac{a_{m} \cos \theta_{m}}{r \tau}+\eta \operatorname{sgn}\left(s_{1}\right)+k_{1} \operatorname{sig}\left(s_{1}\right)^{\alpha}+k_{2} s_{1}\right),
\end{aligned}
$$

where the term $\eta \operatorname{sgn}\left(s_{1}\right)$ is for compensating the disturbance $\dot{g}(t)$, and from (20), $|\dot{g}(t)| \leq L \leq \eta$.

Theorem 12. for the system's equations of states (15) and (30), if we select the sliding mode manifold of (31) and the fast power 
reaching law of (32) and design the guidance law as (35), the system state will reach the sliding mode manifold $s_{1}=0$ within finite time. On $s_{1}=0, s, \dot{s}$ converges to zero after finite time, so that the LOS angle rate and the LOS angular acceleration can converge to zero within finite time.

Proof. Substituting the guidance law of (35) into (33), we can get

$$
\begin{aligned}
\dot{s}_{1}= & \beta \gamma|\dot{s}|^{\gamma-1} \\
& \times\left(-\eta \operatorname{sgn}\left(s_{1}\right)+\dot{g}(t)-k_{1} \operatorname{sig}\left(s_{1}\right)^{\alpha}-k_{2} s_{1}\right) .
\end{aligned}
$$

Construct Lyapunov function:

$$
V_{2}=S_{1}^{2}
$$

Taking the derivative of $V_{2}$, we can get

$$
\begin{aligned}
\dot{V}_{2} & =2 \beta \gamma|\dot{s}|^{\gamma-1} s_{1}\left(-\eta \operatorname{sgn}\left(s_{1}\right)+\dot{g}(t)-k_{1} \operatorname{sig}\left(s_{1}\right)^{\alpha}-k_{2} s_{1}\right) \\
& =2 \beta \gamma|\dot{s}|^{\gamma-1}\left(-\eta\left|s_{1}\right|+s_{1} \dot{g}(t)-k_{1}\left|s_{1}\right|^{\alpha+1}-k_{2} s_{1}^{2}\right) \\
& \leq 2 \beta \gamma|\dot{s}|^{\gamma-1}\left(-\eta\left|s_{1}\right|+\left|s_{1}\right||\dot{g}(t)|-k_{1}\left|s_{1}\right|^{\alpha+1}-k_{2} s_{1}^{2}\right) \\
& \leq 2 \beta \gamma|\dot{s}|^{\gamma-1}\left(-\eta\left|s_{1}\right|+L\left|s_{1}\right|-k_{1}\left|s_{1}\right|^{\alpha+1}-k_{2} s_{1}^{2}\right) \\
& \leq 2 \beta \gamma|\dot{s}|^{\gamma-1}\left(-k_{1}\left|s_{1}\right|^{\alpha+1}-k_{2} s_{1}^{2}\right) \\
& =-\mu V_{2}^{(\alpha+1) / 2}-\lambda V_{2} \\
& \leq 0
\end{aligned}
$$

where $\mu=2 k_{1} \beta \gamma|\dot{s}|^{\gamma-1} \geq 0,0<(\alpha+1) / 2<1, \lambda=2 k_{2}$ $\beta \gamma|\dot{s}|^{\gamma-1} \geq 0$. When $\dot{s} \neq 0$, we can get from Lemma 7 that the system of (15) converges to the sliding mode manifold $s_{1}=0$ within finite time. On $s_{1}=0$, the system state $s, \dot{s}$ converges to zero within finite time; when $\dot{s}=0, s_{1} \neq 0$; according to (31), $s \neq 0$, but $(s \neq 0, \dot{s}=0)$ is a stable equilibrium, which means that $\dot{V}_{2}=0$ cannot maintain. According to the reaching condition of sliding mode, the system will reach and remain the nonsingular terminal sliding mode state $s_{1}=0$; then the system state $s, \dot{s}$ will converge to zero within finite time, which means that the LOS angle rate and the LOS angular acceleration will converge to zero within finite time.

The guidance law designed with (35) is called the fast sliding mode guidance law, or FNTSMG for short.

\section{Numerical Simulation}

Suppose that, in the inertial coordinate system, both the target and the missile are moving in vertical plane. The missile's and the target's initial positions are $x_{m}(0)=0 \mathrm{~km}$, $y_{m}(0)=0 \mathrm{~km}$, and $x_{t}(0)=11.18 \mathrm{~km}, y_{t}(0)=6.5 \mathrm{~km}$, respectively; their initial flight path angles are $\varphi_{m}(0)=30^{\circ}$, $\varphi_{t}(0)=20^{\circ}$, respectively; their flight speeds are $1500 \mathrm{~m} / \mathrm{s}$ and $800 \mathrm{~m} / \mathrm{s}$, respectively; and the guidance distance of the
TABLE 1: miss distances and flight times.

\begin{tabular}{lcccc}
\hline & \multicolumn{2}{c}{ Cosine } & \multicolumn{2}{c}{$\begin{array}{c}\text { Step } \\
\text { maneuvering }\end{array}$} \\
\hline FSMG & $0.039 \mathrm{~m}$ & $18.303(\mathrm{~s})$ & $0.393 \mathrm{~m}$ & $17.298(\mathrm{~s})$ \\
VDSMG & $0.225 \mathrm{~m}$ & $18.304(\mathrm{~s})$ & $0.272 \mathrm{~m}$ & $17.313(\mathrm{~s})$ \\
APNG & $99364 \mathrm{~m}$ & - & $96276 \mathrm{~m}$ & - \\
\hline
\end{tabular}

seeker is $r_{0}=100 \mathrm{~m}$. In the simulation, the parameters of the guidance law are selected as $k_{1}=7, k_{2}=3, \alpha=0.75$, $a_{1}=2, a_{2}=3, \beta=5$, and $\gamma=5 / 3$, and missile's acceleration is limited as $20 \mathrm{~g}, \mathrm{~g}=9.8 \mathrm{~m} / \mathrm{s}^{2}$ is the gravitational constant. The parameters of the observer are selected as $\lambda_{0}=1.1, \lambda_{1}=1.5$, $\lambda_{2}=2, \mu_{0}=3, \mu_{1}=6, \mu_{2}=8$, and $L=100$.

Suppose that the target employs cosine maneuvering of $a_{t}=8 g \cos (\pi t / 4) \mathrm{m} / \mathrm{s}^{2}$ and step maneuvering of $a_{t}=$ $8 \mathrm{gm} / \mathrm{s}^{2}$, respectively. It is necessary to take the place of $\operatorname{sgn}(x)$ with saturation function:

$$
\operatorname{sat}_{\delta}(x)= \begin{cases}1 & x>\delta \\ \frac{x}{\delta} & |x| \leq \delta \\ -1 & x<-\delta\end{cases}
$$

where $\delta$ is small positive constant. Simulation results are introduced as follows.

Case 1. Not considering the dynamic delay of the missile autopilot, we perform simulation comparison between the designed FSMG, VDSMG, and the augmented proportional navigation guidance law [29] $\widehat{a}_{m}=-6 \dot{r} \dot{q}+0.5 z_{1}$ (of APNG for short), where $z_{1}$ is the total disturbance estimated according to the observer of (21). Table 1 shows the miss distances and the flight times; Figures 2-5 illustrate the tracking curves of the LOS angular rate, the LOS angular acceleration, the missile's normal acceleration, the target's maneuvering, and the observer with respect to the target's total disturbance, respectively.

As shown in Table 1, APNG is completely invalid, while both FSMG and VDSMG have small miss distances in any of the two cases, with basically the same flight time. As shown in Figure 2, both FSMG and VDSMG are able to make the LOS angle rate converge to zero rapidly in any of the two cases; in particular, the convergence speed of FSMG is significantly superior to that of VDSMG. At the same time, this figure shows that the LOS angular rate of VDSMG oscillates around zero until it converges to zero, which makes the LOS angular acceleration in Figure 3 and the missile's normal acceleration in Figure 4 oscillate in the first 3 secs. However, both FSMG and VDSMG have sound guidance performance after the first 3 secs and are able to intercept fast targets effectively. We can also see that the missile's acceleration of both FSMG and VDSMG are able to track the target's maneuvering rapidly and without time delay, which confirms the effectiveness of Theorems 8 and 9. On the other side, the LOS angular rate of APNG is divergent in either case that the missile cannot intercept the target. As shown in Figure 5, the designed 

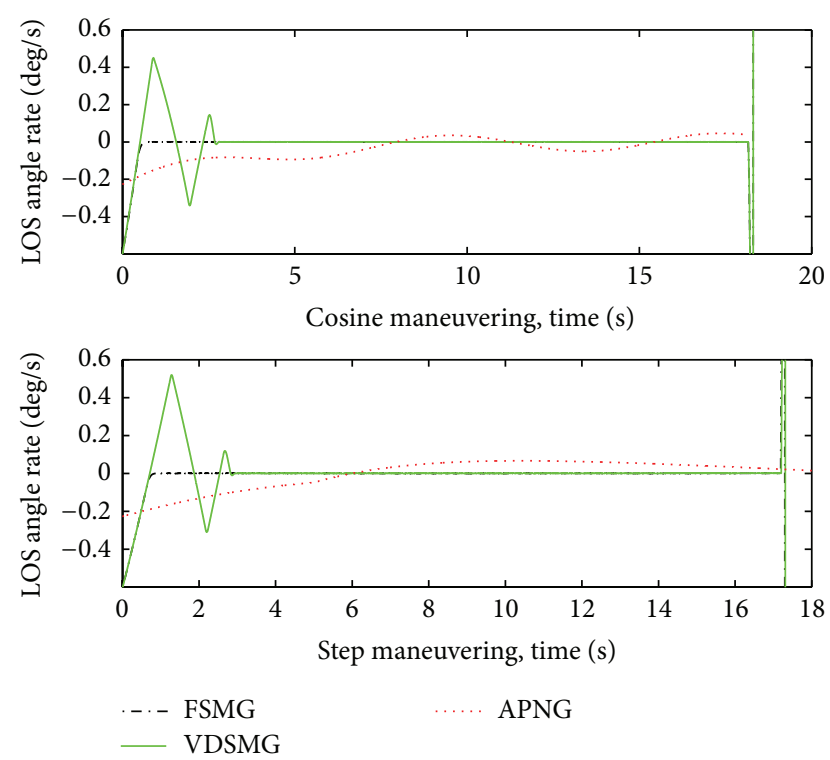

FIGURE 2: Curve of line-of-sight angle rate.
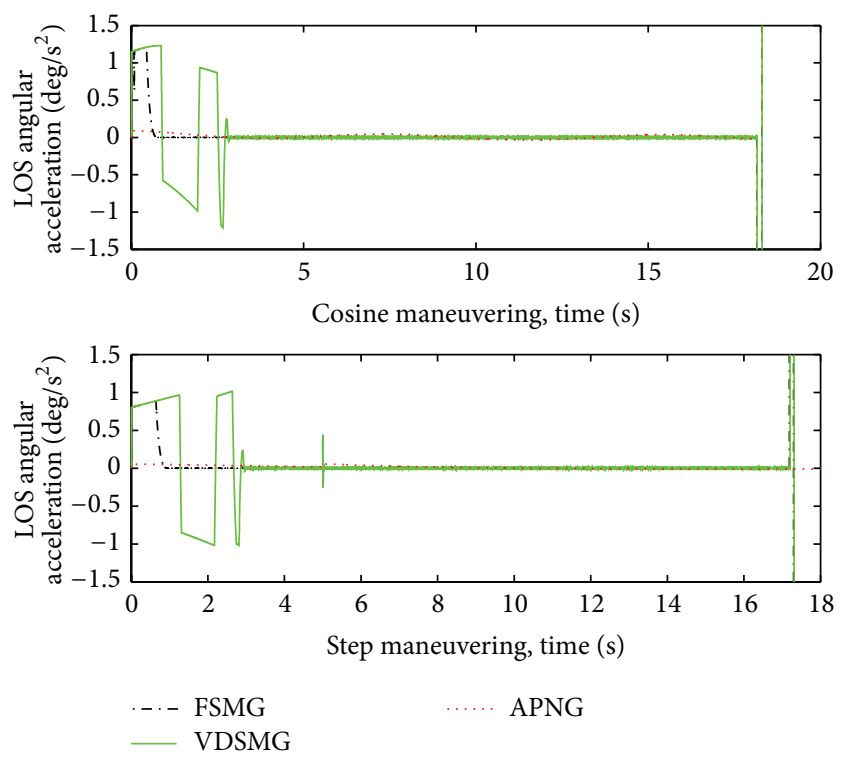

FIgURE 3: Curve of LOS angular acceleration.

observer can track the target's total disturbance rapidly and precisely in less than $0.2 \mathrm{~s}$.

Case 2. Considering that the missile autopilot has first-order delay, we perform a simulation comparison between FSMG, VDSMG, and FNTSMG, where the time constant of missile autopilot is $\tau=0.5$. Table 2 shows the miss distances and flight times; Figures 6-8 illustrate the simulation results.

As shown in Table 2, VDSMG is invalid, while both FSMG and FNTSMG have small miss distances. As shown in Figure 6, the LOS angular rate of VDSMG is immediately divergent, while the LOS angle rate of FSMG oscillates greatly in the first seconds and then tends to zero rapidly, which
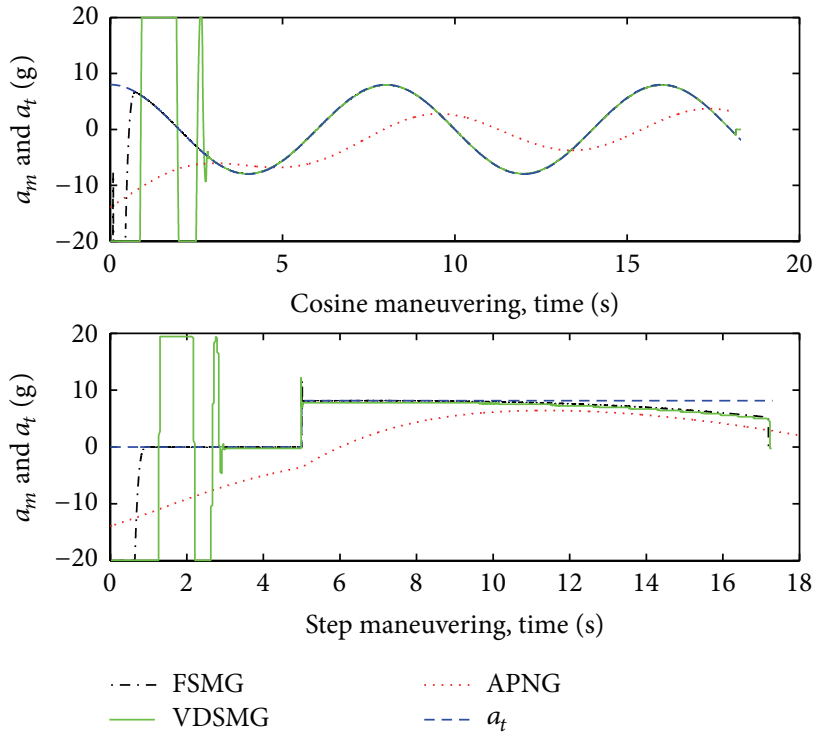

FIGURE 4: Curve of missile normal acceleration and target's acceleration.
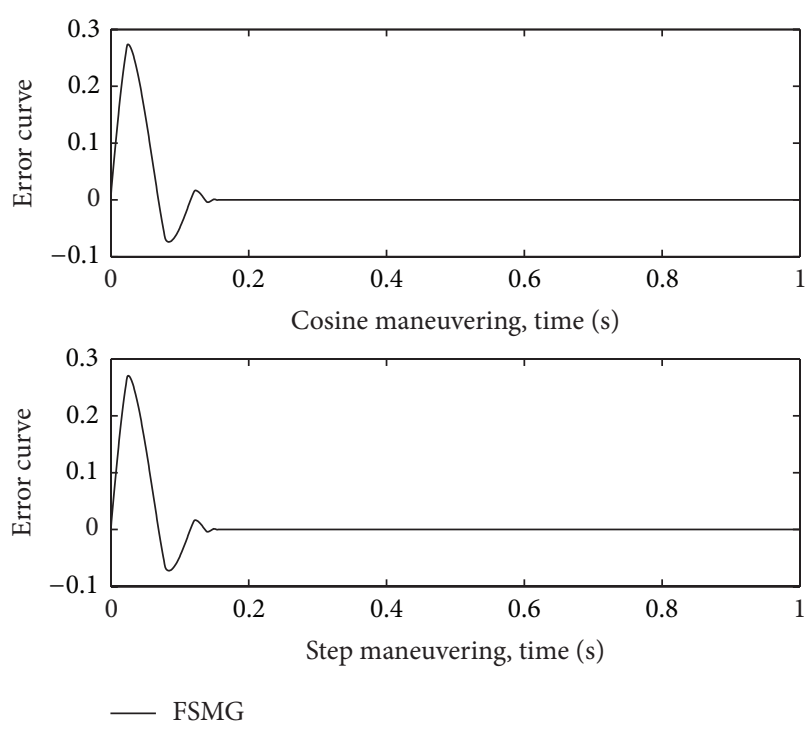

FIGURE 5: Curve of tracking.

TABLE 2: miss distances and flight times.

\begin{tabular}{lcccc}
\hline & \multicolumn{2}{c}{$\begin{array}{c}\text { Cosine } \\
\text { maneuvering }\end{array}$} & \multicolumn{2}{c}{$\begin{array}{c}\text { Step } \\
\text { maneuvering }\end{array}$} \\
\hline FSMG & $0.528 \mathrm{~m}$ & $18.304(\mathrm{~s})$ & $0.857 \mathrm{~m}$ & $17.290(\mathrm{~s})$ \\
VDSMG & $1000.3 \mathrm{~m}$ & $18.166(\mathrm{~s})$ & $445.11 \mathrm{~m}$ & $17.985(\mathrm{~s})$ \\
FNTSMG & $0.127 \mathrm{~m}$ & $18.305(\mathrm{~s})$ & $0.312 \mathrm{~m}$ & $17.285(\mathrm{~s})$ \\
\hline
\end{tabular}

shows that FSMG is more robust than VDSMG. However, the great oscillation in the first seconds results in the oscillation of LOS angular acceleration and missile normal acceleration in Figures 7 and 8, causing the loss of guidance performance. For the designed FNTSMG, as shown in Figures 6-8, both the 

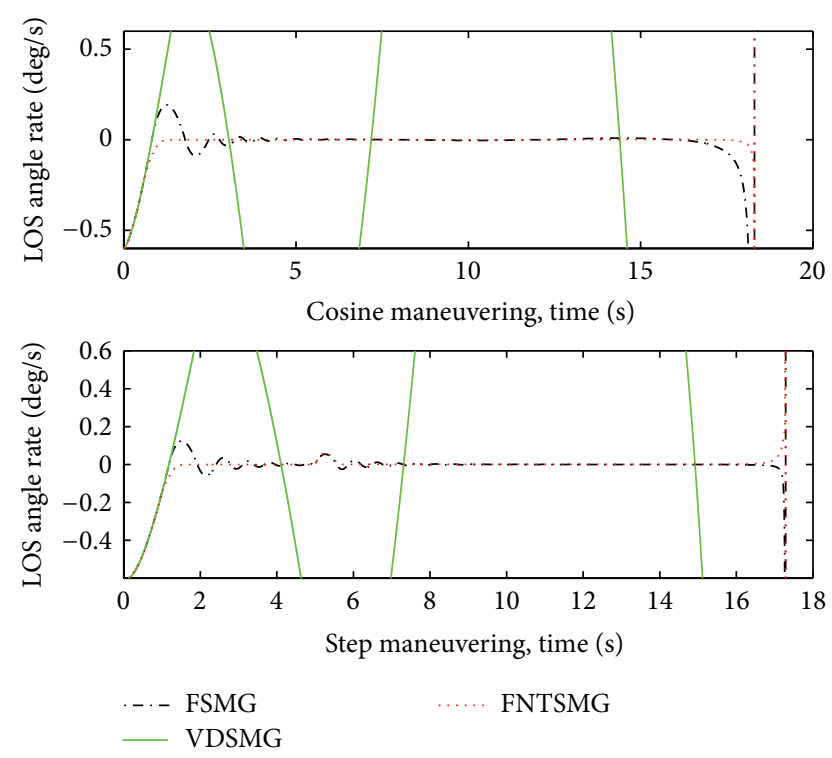

FIGURE 6: Curve of line-of-sight angle rate.
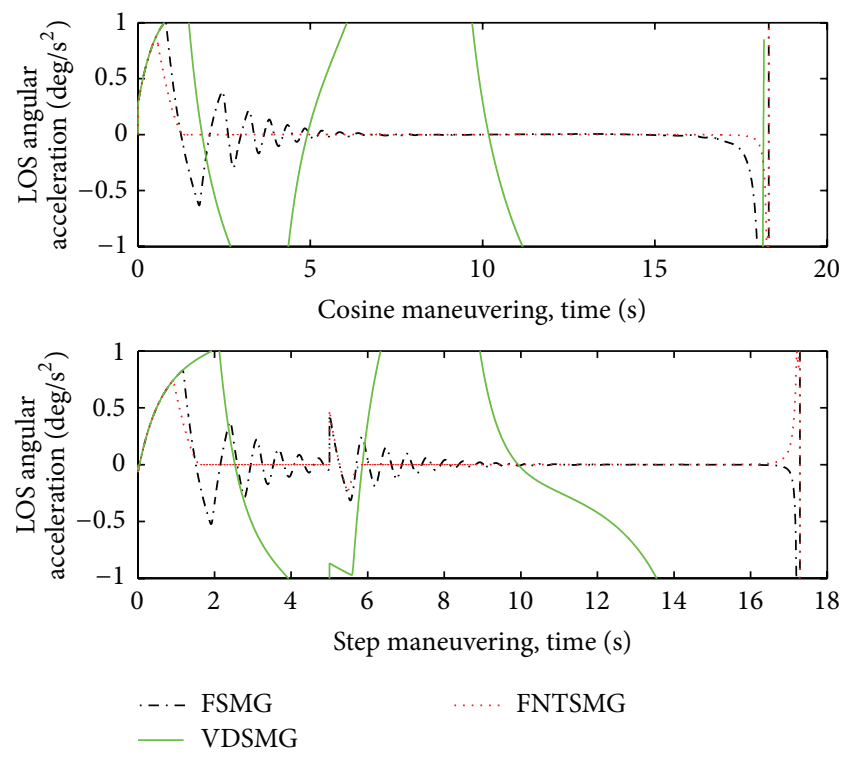

FIGURE 7: Curve of LOS angular acceleration.

TABLE 3: miss distances and flight times.

\begin{tabular}{lllll}
\hline & \multicolumn{2}{c}{ Cosine } & \multicolumn{2}{c}{$\begin{array}{c}\text { Step } \\
\text { maneuvering }\end{array}$} \\
\hline$\tau=0.3$ & $0.031 \mathrm{~m}$ & $18.304(\mathrm{~s})$ & $0.453 \mathrm{~m}$ & $17.290(\mathrm{~s})$ \\
$\tau=0.5$ & $0.127 \mathrm{~m}$ & $18.305(\mathrm{~s})$ & $0.312 \mathrm{~m}$ & $17.287(\mathrm{~s})$ \\
$\tau=0.7$ & $0.696 \mathrm{~m}$ & $18.306(\mathrm{~s})$ & $0.379 \mathrm{~m}$ & $17.285(\mathrm{~s})$ \\
\hline
\end{tabular}

LOS angular rate and the LOS angular acceleration converge to zero rapidly within finite time in both cases. Also the missile's normal acceleration remains in limited range and is able to track the target's maneuvering rapidly, while a time delay exits in the system. All the above confirms the
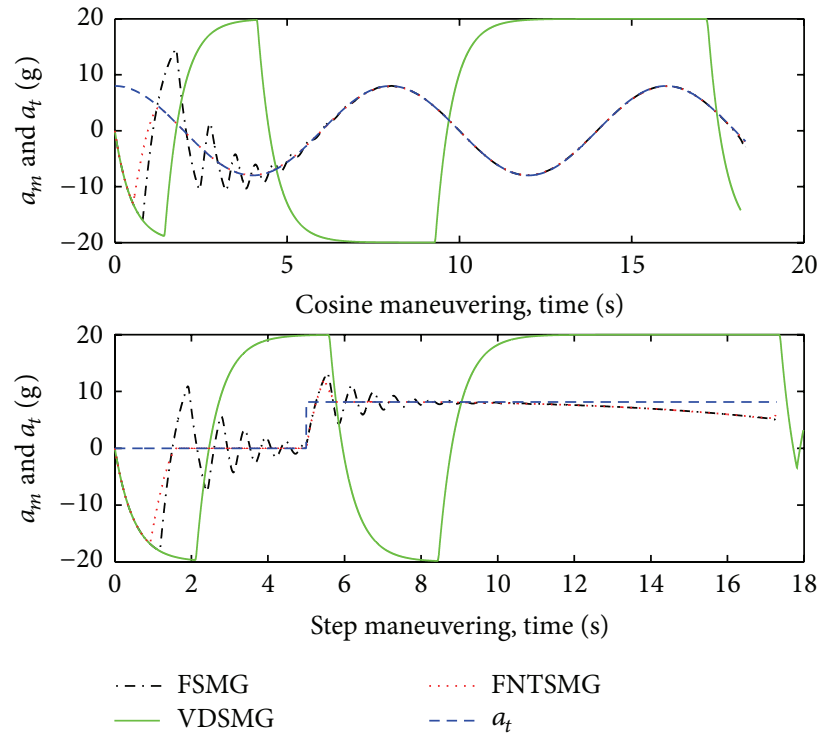

FIGURE 8: Curve of missile normal acceleration and the target's maneuvering.
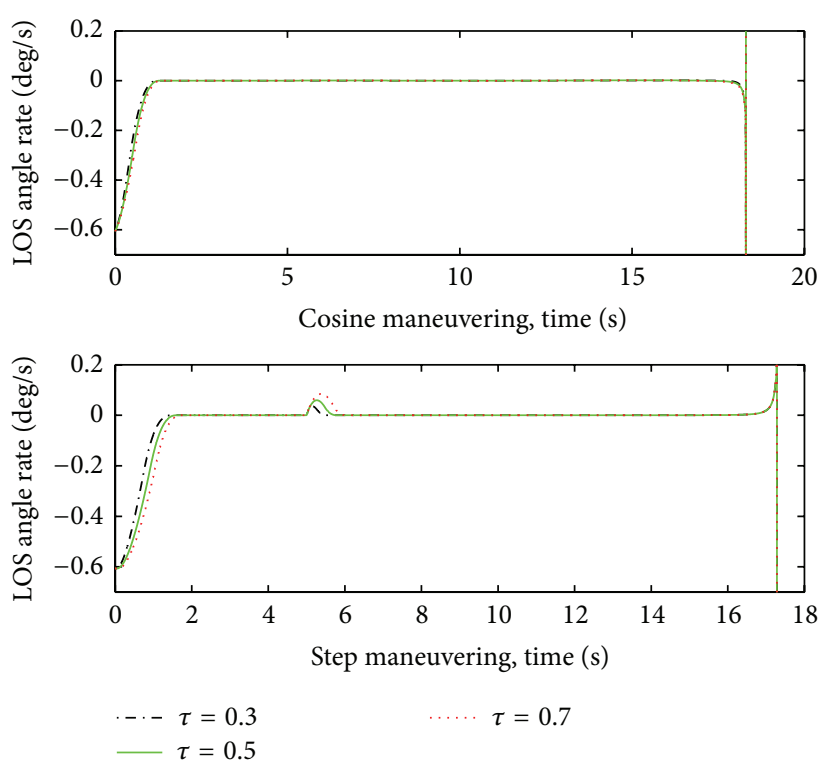

FIGURE 9: Curve of line-of-sight angle rate.

effectiveness of Theorem 12. In one word, it indicates that FNTSMG is able to compensate the influence of the missile's dynamic delay and the target's maneuvering, with a sound and highly precise guidance performance.

Case 3. Considering that the missile autopilot has firstorder delay, we perform a simulation to show the validity of FNTSMG. The time constant of the missile autopilot is chosen as $\tau=0.3,0.5,0.7$, respectively. Table 3 shows the miss distances and flight times. Figures 9-11 illustrate the simulation results.

As shown in Table 3, when the time constant of the missile autopilot varies, the corresponding miss distance 

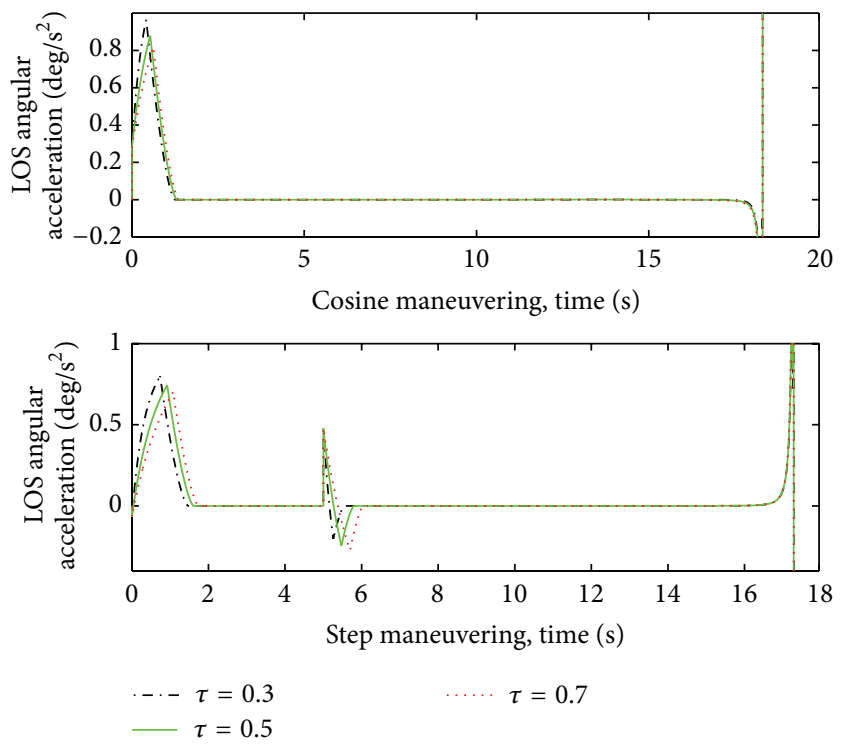

FIGURE 10: Curve of LOS angular acceleration.
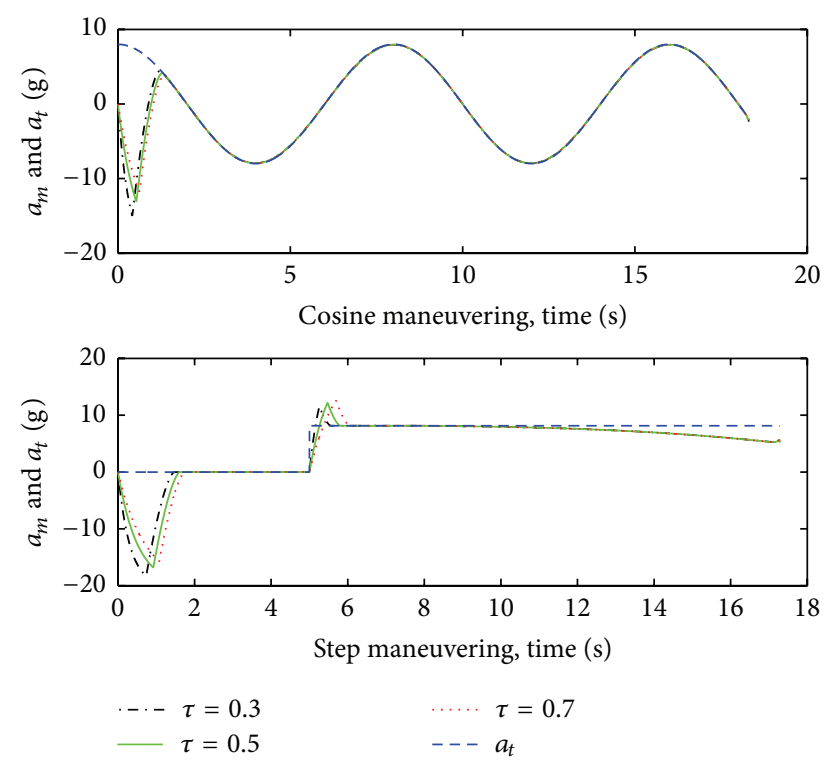

FIGURE 11: Curve of missile normal acceleration and the target's maneuvering.

remains small and flight time almost the same as each other, which shows the robustness of the proposed guidance law. From Figures 9 and 10, it can be seen that both the LOS angular rate and the LOS angular acceleration converge to zero rapidly. Figure 11 illustrates that when the target is escaping substantially under two different cases, the missile's normal acceleration is able to track the target's maneuvering rapidly and remains in limited range, which confirms the effectiveness of Theorem 12.

\section{Conclusions}

For the target's escape maneuvering in the missile interception system, we apply finite-time convergent nonhomogeneous disturbance observer to track and observe the target's unknown disturbance. When not considering the dynamic delay of missile autopilot, two guidance laws are designed, namely, FSMG and VDSMG. According to the result of simulation comparison, both methods have a precision of guidance significantly higher than that of APNG which is already known in the field in different cases of the target's maneuvering, and the designed guidance laws can intercept the maneuvering target rapidly with a high precision within finite time. Meanwhile, the result of simulation comparison indicates that FSMG is superior to VDSMG with respect to convergence time and mitigating the chattering of the sliding mode.

Considering the missile's dynamic delay, we select highorder nonsingular terminal sliding mode and design a FNTSMG which is able to compensate the dynamic delay of missile autopilot and the target's maneuvering. From the simulation results, it can be seen that the guidance law can always make sure a performance of high precision and stability under different dynamic time delays, which shows the efficiency and robustness of the proposed guidance law. Future works include the design of the guidance law that can deal with the dynamic time delay and impact angle constraint at the same time, while the whole system evolves in 3-dimensional space.

\section{Nomenclature}

$a_{m}:$ Missile's normal acceleration

$a_{t}$ : Target's normal acceleration

$V_{m}:$ Missile's speed

$V_{t}:$ Target's speed

$r$ : Relative distance between the missile and the target

$\dot{r}$ : Relative speed between the missile and the target

$q$ : Line-of-sight (LOS) angle

$\dot{q}: \quad$ Line-of-sight (LOS) angular rate

$\varphi_{m}:$ Missile's flight path angle

$\varphi_{t}:$ Target's flight path angle

$\theta_{m}:$ Advance angle of missile

$\theta_{t}$ : Advance angle of target

$a_{m c}$ : The command to the autopilot

$s: \quad$ Sliding mode.

\section{Conflict of Interests}

The authors declare that there is no conflict of interests regarding the publication of this paper.

\section{Acknowledgments}

The authors would like to acknowledge the financial support provided by the Foundation for Creative Research Groups of the National Natural Science Foundation of China under Grant no. 61021002, the Natural Science Foundation of Heilongjiang Province, China (no. A201410), and the Project of Education Department of Heilongjiang Province, China (no. 12541251). 


\section{References}

[1] B. S. Chen, Y. Y. Chen, and C. L. Lin, "Nonlinear fuzzy guidance law with saturation of actuators against maneuvering targets," IEEE Transactions on Control Systems Technology, vol. 10, no. 6, pp. 769-779, 2002.

[2] C. D. Yang and H. Y. Chen, "Three-dimensional nonlinear $H_{\infty}$ guidance law," International Journal of Robust and Nonlinear Control, vol. 11, no. 2, pp. 109-129, 2001.

[3] C.-S. Shieh, "Tunable $H_{\infty}$ robust guidance law for homing missiles," IEE Proceedings: Control Theory and Applications, vol. 151, no. 1, pp. 103-108, 2004.

[4] D. Zhou, C. Mu, and T. Shen, "Robust guidance law with $L_{2}$ gain performance," Transactions of the Japan Society for Aeronautical and Space Sciences, vol. 44, no. 144, pp. 82-88, 2001.

[5] N. Boëly and R. M. Botez, "New approach for the identification and validation of a nonlinear F/A-18 model by use of neural networks," IEEE Transactions on Neural Networks, vol. 21, no. 11, pp. 1759-1765, 2010.

[6] T. L. Grigorie and R. M. Botez, "New adaptive controller method for SMA hysteresis modelling of a morphing wing," Aeronautical Journal, vol. 114, no. 1151, pp. 1-13, 2010.

[7] J. Moon, K. Kim, and Y. Kim, "Design of missile guidance law via variable structure control," Journal of Guidance, Control, and Dynamics, vol. 24, no. 4, pp. 659-664, 2001.

[8] C.-M. Lin and C.-F. Hsu, "Guidance law design by adaptive fuzzy sliding-mode control," Journal of Guidance, Control, and Dynamics, vol. 25, no. 2, pp. 248-256, 2002.

[9] T. Shima, M. Idan, and O. M. Golan, "Sliding-mode control for integrated missile autopilot guidance," Journal of Guidance, Control, and Dynamics, vol. 29, no. 2, pp. 250-260, 2006.

[10] Y. B. Shtessel and C. H. Tournes, "Integrated higher-order sliding mode guidance and autopilot for dual-control missiles," Journal of Guidance, Control, and Dynamics, vol. 32, no. 1, pp. 79-94, 2009.

[11] Y. B. Shtessel, I. A. Shkolnikov, and A. Levant, "Smooth secondorder sliding modes: missile guidance application," Automatica, vol. 43, no. 8, pp. 1470-1476, 2007.

[12] L. Wang, Y. Sheng, and X. Liu, "A novel adaptive highorder sliding mode control based on integral sliding mode," International Journal of Control, Automation and Systems, vol. 12, no. 3, pp. 459-472, 2014.

[13] Y. Feng, X. Yu, and Z. Man, "Non-singular terminal sliding mode control of rigid manipulators," Automatica, vol. 38, no. 12, pp. 2159-2167, 2002.

[14] S. Yu, X. Yu, B. Shirinzadeh, and Z. Man, "Continuous finitetime control for robotic manipulators with terminal sliding mode," Automatica, vol. 41, no. 11, pp. 1957-1964, 2005.

[15] Z. Zhu, D. Xu, J. Liu, and Y. Xia, "Missile guidance law based on extended state observer," IEEE Transactions on Industrial Electronics, vol. 60, no. 12, pp. 5882-5891, 2013.

[16] Y. Xia, Z. Zhu, and M. Fu, "Back-stepping sliding mode control for missile systems based on an extended state observer," IET Control Theory \& Applications, vol. 5, no. 1, pp. 93-102, 2011.

[17] H. Yan and H. B. Ji, "Guidance laws based on input-tostate stability and high-gain observers," IEEE Transactions on Aerospace and Electronic Systems, vol. 48, no. 3, pp. 2518-2529, 2012.

[18] K. Ma, H. K. Khalil, and Y. Yao, "Guidance law implementation with performance recovery using an extended high-gain observer," Aerospace Science and Technology, vol. 24, no. 1, pp. 177-186, 2013.
[19] A. Levant, "Non-homogeneous finite-time-convergent differentiator," in Proceedings of the 48th IEEE Conference on Decision and Control held jointly with the 28th Chinese Control Conference (CDC/CCC '09), pp. 8399-8404, Shanghai, China, December 2009.

[20] L. Peng, P. Xuefeng, M. Jianjun, and T. Shuai, "Non-homogeneous disturbance observer-based second order sliding mode control for a tailless aircraft," in Proceeding of the Chinese Automation Congress (CAC '13), pp. 120-125, Changsha, China, November 2013.

[21] R. H. Chen, J. L. Speyer, and D. Lianos, "Game-theoretic homing missile guidance with autopilot lag," in Proceedings of the AIAA Guidance, Navigation, and Control Conference and Exhibit, pp. 2132-2145, Hilton Head Island, SC, USA, August 2007.

[22] R. H. Chen, J. L. Speyer, and D. Lianos, "Optimal intercept missile guidance strategies with autopilot lag," Journal of Guidance, Control, and Dynamics, vol. 33, no. 4, pp. 1264-1272, 2010.

[23] Z. Zhang, S. Li, and S. Luo, "Composite guidance laws based on sliding mode control with impact angle constraint and autopilot lag," Transactions of the Institute of Measurement and Control, vol. 35, no. 6, pp. 764-776, 2013.

[24] P. P. Qu and D. Zhou, "A dimension reduction observer-based guidance law accounting for dynamics of missile autopilot," Proceedings of the Institution of Mechanical Engineers G: Journal of Aerospace Engineering, vol. 227, no. 7, pp. 1114-1121, 2013.

[25] S. Sun, D. Zhou, and W.-T. Hou, "A guidance law with finite time convergence accounting for autopilot lag," Aerospace Science and Technology, vol. 25, no. 1, pp. 132-137, 2013.

[26] S. P. Bhat and D. S. Bernstein, "Finite-time stability of continuous autonomous systems," SIAM Journal on Control and Optimization, vol. 38, no. 3, pp. 751-766, 2000.

[27] A. Levant, "Higher-order sliding modes, differentiation and output-feedback control," International Journal of Control, vol. 76, no. 9-10, pp. 924-941, 2003.

[28] Y. B. Shtessel, I. A. Shkolnikov, and A. Levant, "Guidance and control of missile interceptor using second-order sliding modes," IEEE Transactions on Aerospace and Electronic Systems, vol. 45, no. 1, pp. 110-124, 2009.

[29] J. Z. Ben-Asher and I. Yaesh, "Optimal guidance with reduced sensitivity to time-to-go estimation errors," Journal of Guidance, Control, and Dynamics, vol. 20, no. 1, pp. 158-163, 1997. 

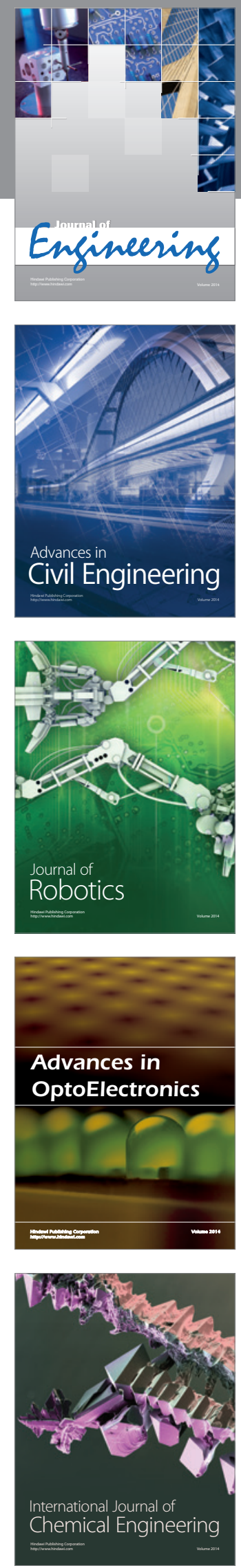

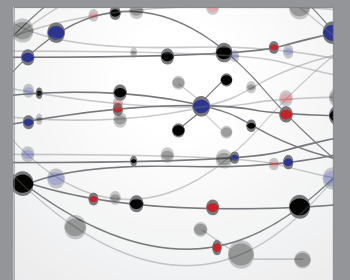

The Scientific World Journal
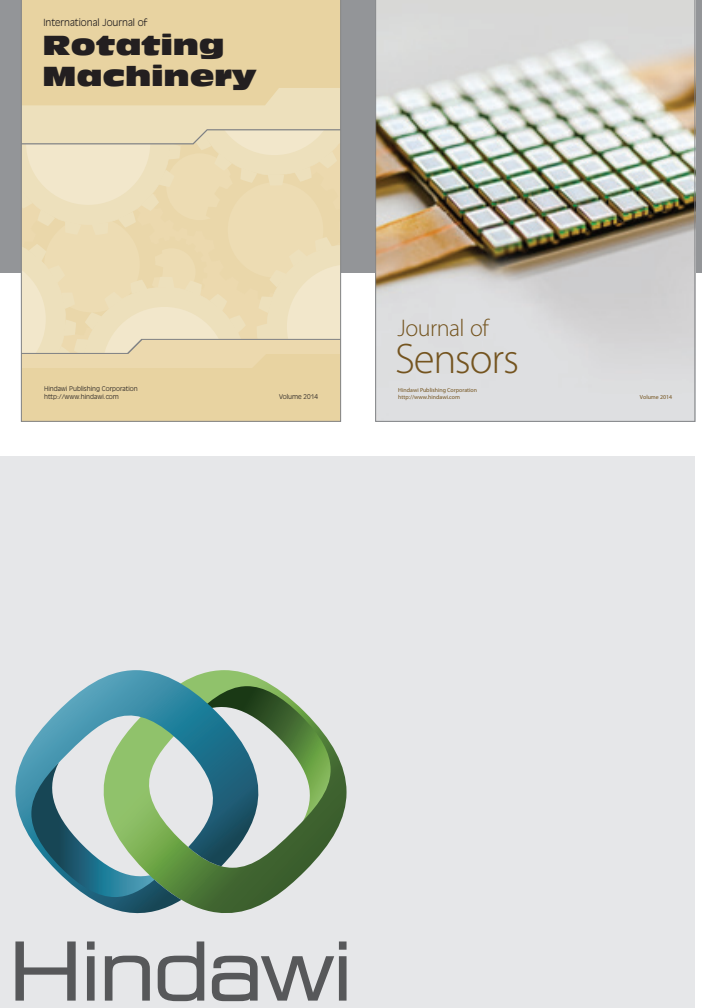

Submit your manuscripts at http://www.hindawi.com
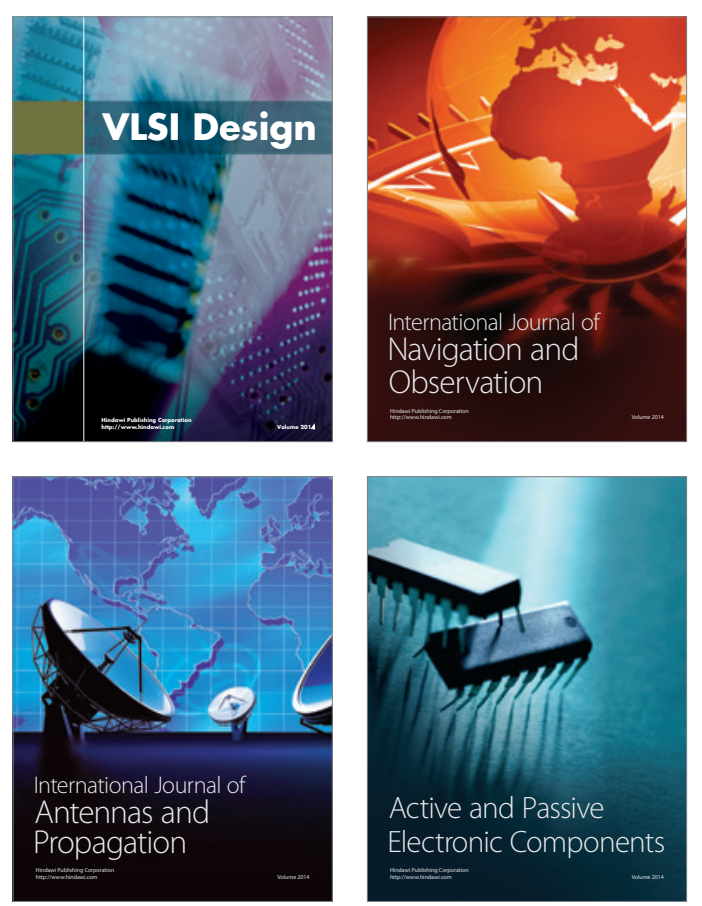
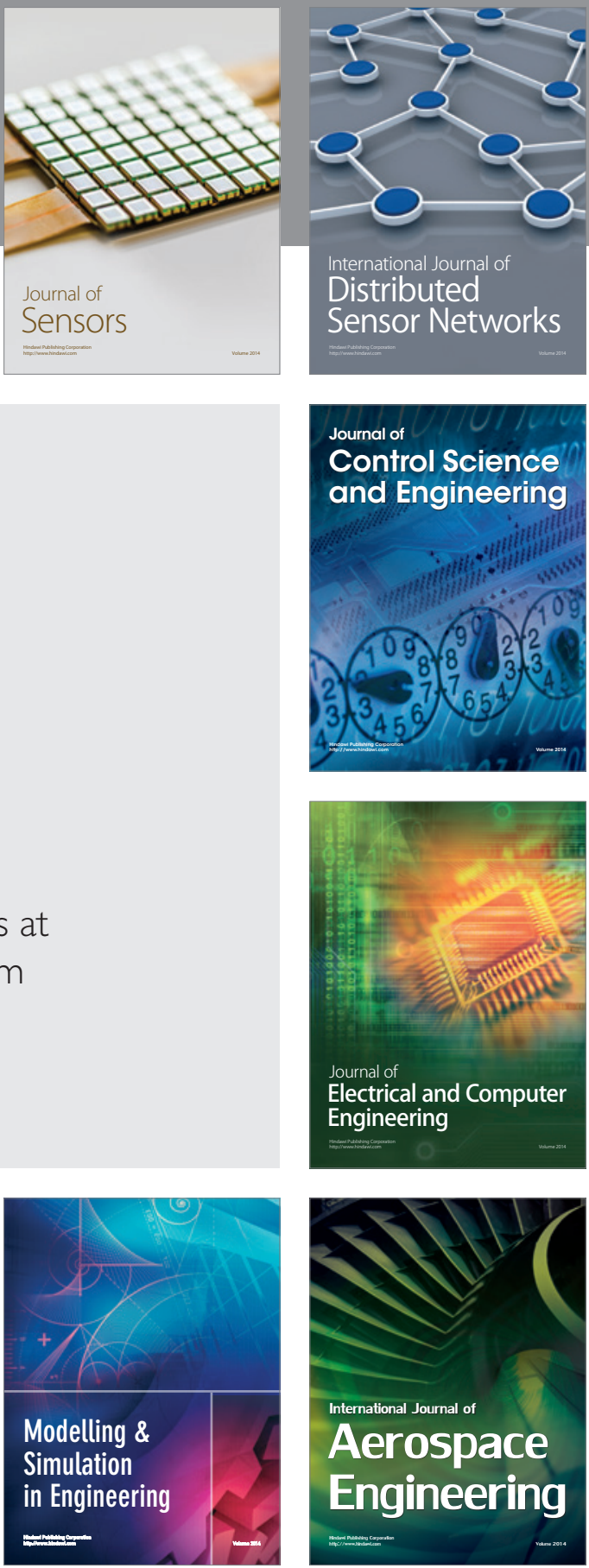

Journal of

Control Science

and Engineering
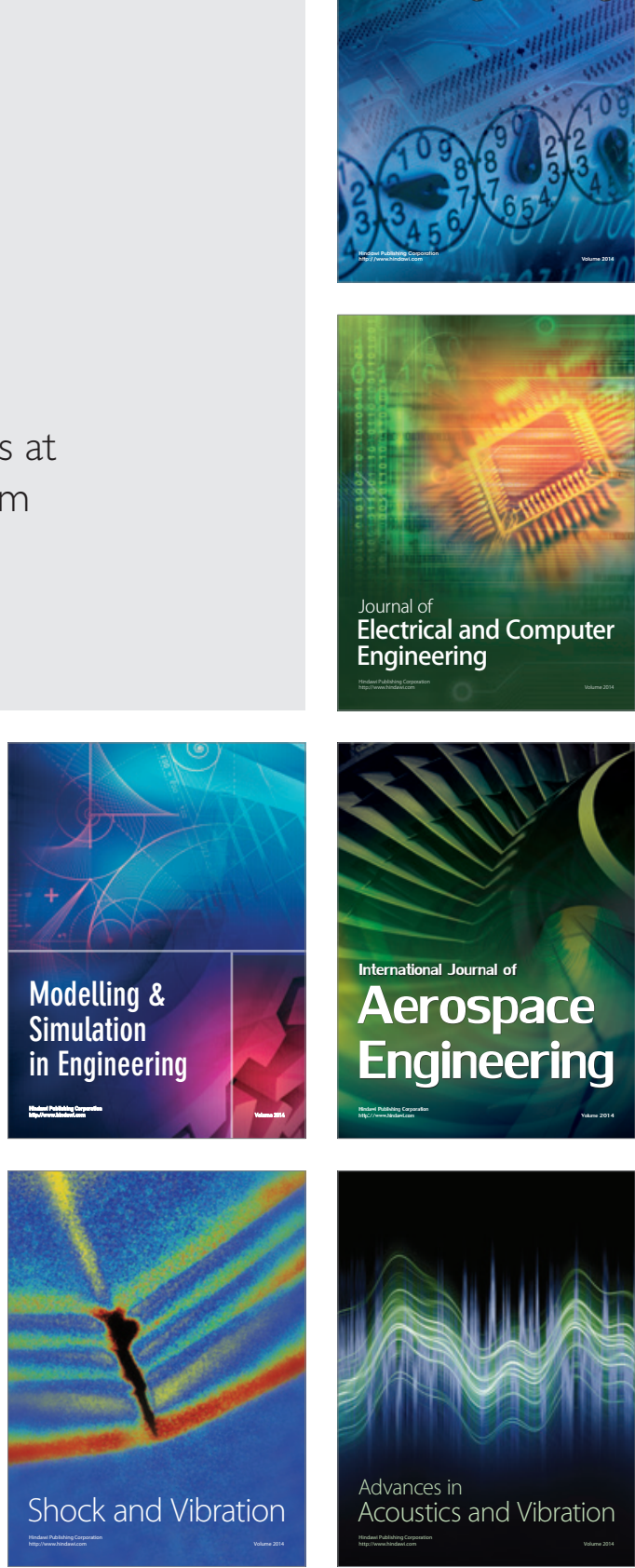